\title{
Crystal structure and Hirshfeld surface analysis of 2-(1H-indol-3-yl)ethanaminium acetate hemihydrate. Corrigendum
}

\author{
Balakrishnan Rajeswari, ${ }^{a}$ Radhakrishnan Santhi, ${ }^{a}$ Palaniyappan Sivajeyanthi ${ }^{\mathbf{b}}$ and \\ Kasthuri Balasubramani ${ }^{\text {b* }}$
}

aPG and Research Department of Chemistry, Seethalakshmi Ramaswamy College (Affiliated to Bharathidasan University), Tiruchirappalli-2, Tamil Nadu, India, and ${ }^{\mathbf{b}}$ Department of Chemistry, Government Arts College (Affiliated to Bharathidasan University), Thanthonimalai, Karur 639 005, Tamil Nadu, India. *Correspondence e-mail: manavaibala@gmail.com

In the paper by Rajeswari et al. [Acta Cryst. (2019), E75, 451-455], the affiliations were not given correctly.

The affiliations in the paper by Rajeswari et al. (2019) are incorrect and should be ${ }^{\mathrm{a}} \mathrm{PG}$ and Research Department of Chemistry, Seethalakshmi Ramaswamy College (Affiliated to Bharathidasan University), Tiruchirappalli-2, Tamil Nadu, India and ${ }^{\mathrm{b}}$ Department of Chemistry, Government Arts College (Affiliated to Bharathidasan University), Thanthonimalai, Karur 639 005, Tamil Nadu, India', as given above.

\section{References}

Rajeswari, B., Santhi, R., Sivajeyanthi, P. \& Balasubramani, K. (2019). Acta Cryst. E75, 451-455. 